Military Technical College Kobry El-Kobbah, Cairo, Egypt

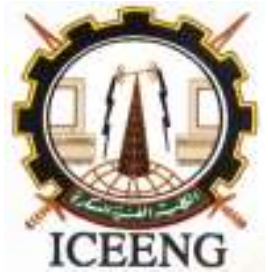

\author{
11th International Conference \\ on Electrical Engineering \\ ICEENG 2018
}

\title{
Application of Chaotic Sequence in Underwater Communication under Effect of Gaussian Mixture Noise
}

\author{
By
}

Hany A. A. Mansour*

\begin{abstract}
The communications under the shallow water are affected by the impulsive components of the ambient noise, in which its statistics does not follow the normal Gaussian distribution. Since most of the communication receivers are designed to receive the Gaussian noise signals, its Performance degrade in the presence of non-Gaussian noise. The noise with Gaussian Mixture (GM) distribution can be considered as one of the most popular non-Gaussian noise that is used to model the UWA, due to its universal approximation properties. However, there were a little researches investigate and present an analytical analysis of the spread spectrum error probability in the UWA with the GM noise. In this paper, the traditional Chaotic Sequence Code (CSC) is applied and verified as a spreading code for UWA channel with GM noise. The error performance is presented and derived analytically with and without the spreading process. The results show that applying the CSC significantly improves the performance in the UWA with the GM noise.
\end{abstract}

\section{Key Words}

Underwater acoustic communication, Gaussian distribution, non-Gaussian noise, Gaussian mixture noise.

\section{I- INTRODUCTION}

Although PN code has attractive properties, however it has also many drawbacks such as the limitation of the number of such sequences, in addition to its periodicity feature, which make the intercepted signal is predictable and is reconstructed by linear regression which leads to security limitations. One of the alternative solutions is the chaotic code, which has a lot of attractive properties over the conventional PN sequences, especially from the security point of view. It is non-periodic, wide band, and more difficult to

\footnotetext{
* Egyptian Armed Forces.
} 
predict and to reconstruct. Moreover, Chaos is a deterministic, random-like process found in non-linear dynamical system, which is non-converging and bounded [1]. It has also a very sensitive dependence upon its initial condition and parameters that gives the availability to generate an infinite number of spreading codes [2]. These properties make chaotic codes more difficult to intercept, and more secured to decode the information spreaded upon them [3].

The central limit theorem (CLT) assumes that the sum of $\mathbf{K}$ independent and identically distributed (i.i.d.) random variables with finite variance converges to a Gaussian distribution as $\mathbf{K} \rightarrow \infty$ [4]. According to this assumption, The Performance of a communication system depends on the statistical characteristics of channel noise which is mostly assumed to be Gaussian in nature. In the case of the underwater acoustic (UWA) communication channel, there are also several sources of random noise, such as: merchant ships, underwater explosives, biological sources, waves, thermal noise, and surface agitation [5]-[7]. These sources of noises can be represented accumulatively by the Gaussian distribution. Nevertheless, in the UWA channel, it is found that for a given frequency band of operation, there are a specific noise sources or even one dominant source has a dominant effect for overall cumulative noise [8]. In this case, the CLT can't be assumed and applied, and consequently the channel can't be approximated to the traditional Gaussian distribution.

Alternatively, there was many several non-Gaussian models proposed in many literatures [9] - [14]. The Middleton model can be considered the first model proposed to simulate the non-Gaussian UWA channel; however its main drawback is that its density function is represented by an infinite series of weighted Gaussian PDF's [10]. The Generalized Gaussian (GG) model is another model represented in [9] and [11], which describes and approximates a wide range of super-Gaussian to sub-Gaussian densities. The main limitation of the GG model is that its density function does not have a closed form except for special cases, and thus the density function is approximated by the Cauchy-Gaussian mixture model [13], or Gaussian Mixture model [14].

Regarding to the Gaussian Mixture (GG) model, it can be defined as a parametric Probability density function which represented as a weighted sum of Gaussian component densities [15]. Due to its universal properties, the GG distribution becomes very popular in modeling the UWA channel noise. It is found that there are several UWA applications such as cooperative OFDM system [16], 3-D localization of UWA sources [17], and ROV navigation [18] apply the GM statistics as a channel noise model.

The new contribution of this paper is to represent an analytical analysis of the error probability for UWA channel represented by the GM noise considering a DSSS system. The CSC is considered as a spreading code in the DSSS system with different lengths. The paper presents also the performance comparison and the performance evaluation of the proposed system, showing the improvement of applying the CSC in the UWA-GM channel. The results show that the analytical results are completely coincident with the simulation results, which gives an indication that both the analytical and simulation results are completely correct.

\section{II-ANALYTICAL ANALYSIS}

In this section, the analytical analysis is presented and the closed form of the probability of error for both the BPSK, and the DS-BPSK is obtained in presence of the GM noise.

\section{A. GM General Background}

The Gaussian Mixture Model (GMM) can be defined as a parametric probability density function represented by a weighted sum of Gaussian component densities. GMMs are commonly used as a parametric model of the probability distribution of continuous measurements or features in a biometric system, such as UWA noise, and vocal-tract related spectral features in a speaker recognition system [15]. 
The Gaussian mixture model is a weighted sum of $\mathbf{K}$ component Gaussian densities which can be represented in a general form as shown in (1):

$$
p(X / \lambda)=\sum_{i=1}^{K} \epsilon_{i} g\left(X / \mu_{i}, \sigma_{i}\right)
$$

WhereX is a D-dimensional continuous-valued data vector (i.e. measurement or features), $\boldsymbol{\epsilon}_{\mathbf{i}}, \mathbf{i}=$ $\mathbf{1}, \ldots, \mathbf{M}$, are the mixture weights (or mixture coefficients), and $\mathbf{g}\left(\mathbf{X} / \boldsymbol{\mu}_{\mathbf{i}}, \boldsymbol{\sigma}_{\mathbf{i}}\right), \mathbf{i}=\mathbf{1}, \ldots, \mathbf{M}$, are the component Gaussian densities. Each component density is a D-variate Gaussian function takes the form of:

$$
g\left(X / \mu_{i}, \sigma_{i}\right)=\frac{1}{(2 \pi)^{\frac{D}{2}}\left|\sigma_{i}\right|^{\frac{1}{2}}} \exp \left\{-\frac{1}{2}\left(X-\mu_{i}\right)^{\prime}{\sigma_{i}}^{-1}\left(X-\mu_{i}\right)\right\}
$$

With mean vector $\boldsymbol{\mu}_{\mathbf{i}}$ and covariance matrix $\boldsymbol{\sigma}_{\mathbf{i}}$. The mixture weights satisfy the constraint that $\sum_{\mathbf{i}=\mathbf{1}}^{\mathbf{K}} \boldsymbol{\epsilon}_{\mathbf{i}}=\mathbf{1}$, and $\mathbf{0} \leq \boldsymbol{\epsilon}_{\mathbf{i}} \leq \mathbf{1}$. For a stochastic noise process $\mathbf{N}$ with $\mathbf{K}$-component GM statistics, the pdf can be given by

$$
f_{G M}(n)=\sum_{i=1}^{K} \epsilon_{i} N\left(\mu_{i}, \sigma_{i}\right)=\sum_{i=1}^{K} \frac{\epsilon_{i}}{\sqrt{2 \pi} \sigma_{i}} \exp \left\{\frac{-\left(n-\mu_{i}\right)}{\sqrt{2} \sigma_{i}}\right\}^{2}
$$

In which the mean $\boldsymbol{\mu}_{\mathbf{G M}}$, and the variance $\boldsymbol{\sigma}_{\mathbf{G M}}$ of the resultant noise process can be given according to the following form:

$$
\mu_{G M}=\sum_{i=1}^{K} \epsilon_{i} \mu_{i}, \quad \sigma_{G M}=\sum_{i=1}^{K} \epsilon_{i} \sigma_{i}^{2}
$$

Fig. 1 presents an example of the probability density function for four different Gaussian noises, and their equivalent Gaussian Mixture. The Gaussian noises components presented in this example have the statistics shown in table I:

TABLE I

PARAMETERS OF THE GAUSSIAN NOISES

\begin{tabular}{cccc}
\multicolumn{4}{c}{ PARAMETERS OF THE GAUSSIAN NOISES } \\
\cline { 2 - 4 } & $\epsilon$ & $\mathrm{M}$ & $\Sigma$ \\
GAUSSIAN & 0.1 & 0 & 0.84 \\
NOISE 1 & & & \\
GAUSSIAN & 0.35 & 2.50 & 3.71 \\
NOISE 2 & & & \\
GAUSSIAN & 0.4 & 5.25 & 1.93 \\
NOISE 3 & & & \\
GAUSSIAN & 0.15 & 6.50 & 2.45 \\
NOISE 4 & &
\end{tabular}

It is clear from the figure that the resultant GM noise has a distorted Gaussian pdf with equivalent mean $\boldsymbol{\mu}_{\mathrm{GM}}=3.95$, and equivalent standard deviation $\boldsymbol{\sigma}_{\mathrm{GM}}=\mathbf{2 . 6 9 8 6}$ calculated according to (4). It is shown in [16] that the GM distribution can approximate any arbitrarily shaped non-Gaussian densities based on the Weiner's approximation theorem, which gives its name of universal approximation. This attribute makes the GM noise very popular in modeling the UWA channel. 


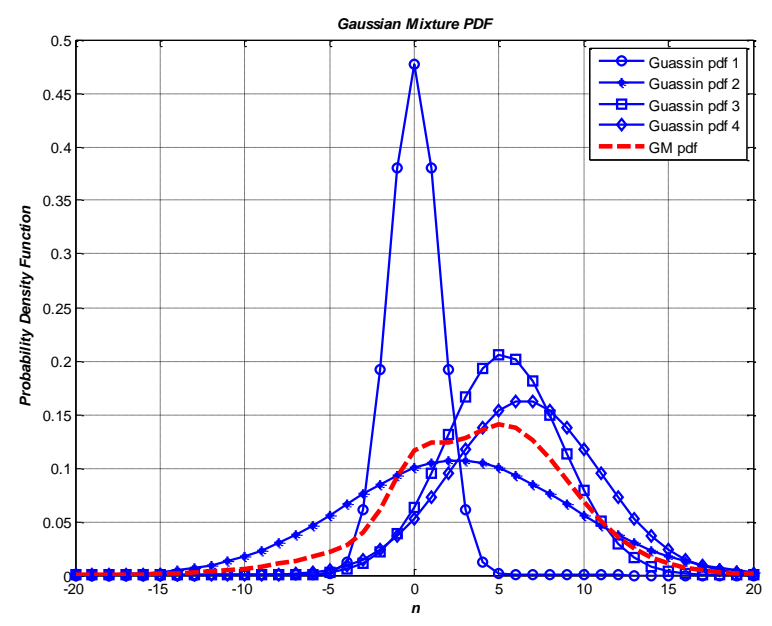

Figure 1 the probability density functions of four different Gaussian noises, and their equivalent Gaussian mixture noise.

In case of existing a low-power nearly Gaussian background noise randomly spiked with high amplitude short duration pulses, the noise density in such cases becomes a mixture of two Gaussian components. These two components have densities weighted according to the impact of each component on the overall ambient noise. The total GM noise can be modeled in this case by the empirical $\boldsymbol{\epsilon}$-mixture or $\boldsymbol{\epsilon}$ contaminated GM density function according to the following expression shown in (5):

$$
f_{G M}(n)=\frac{(1-\epsilon)}{\sqrt{2 \pi} \sigma_{b}} \exp \left\{\frac{-\left(n-\mu_{b}\right)}{\sqrt{2} \sigma_{b}}\right\}+\frac{\epsilon}{\sqrt{2 \pi} \sigma_{i}} \exp \left\{\frac{-\left(n-\mu_{i}\right)}{\sqrt{2} \sigma_{i}}\right\}
$$

Where $\boldsymbol{\mu}_{\mathbf{b}}, \boldsymbol{\sigma}_{\mathbf{b}}$ represent the mean and the standard deviation of the nearly Gaussian noise component, and $\boldsymbol{\mu}_{\mathbf{i}}, \boldsymbol{\sigma}_{\mathbf{i}}$ represents mean and the standard deviation of the impulsive noise where $\boldsymbol{\sigma}_{\mathbf{b}} \leq \mathbf{1} \leq \boldsymbol{\sigma}_{\mathbf{i}}$. In this case, $\boldsymbol{\epsilon} \in[\mathbf{0}, \mathbf{1}]$ controls the contribution from each component, and typical values of $\boldsymbol{\epsilon}$ ranges from 0.01 to 0.3. Fig. (2) shows the resultant pdf of the GM noise in this case for the following parameters, $\boldsymbol{\mu}_{\mathbf{b}}=\mathbf{0}, \boldsymbol{\mu}_{\mathbf{i}}=$ $2.65, \sigma_{\mathrm{b}}=0.765, \sigma_{\mathrm{i}}=2.352$, and $\epsilon=0.25$.

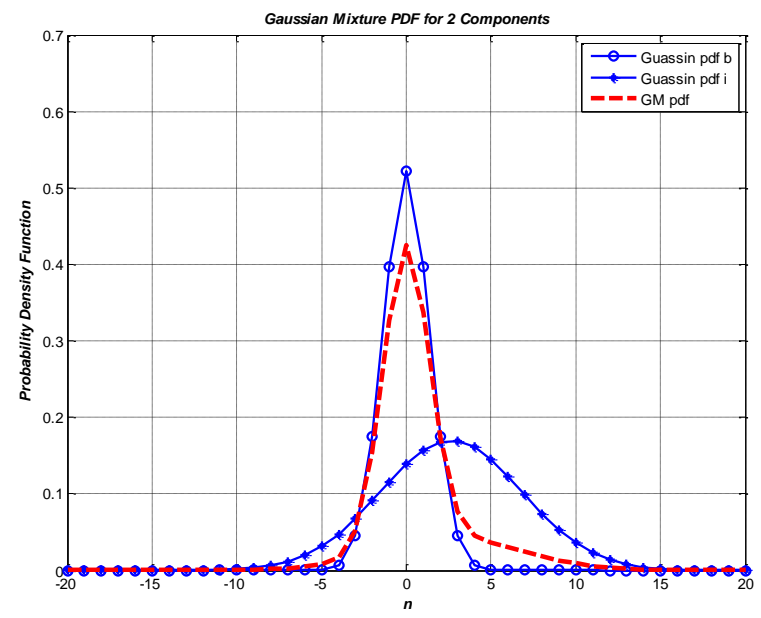

Figure 2 pdf of the GM noise in case of 2 dominant noise components. 


\section{B. Error Probability Analysis}

In this subsection, a closed form for the error probability is analyzed and derived for the UWA communication system in both the cases of the spreading and non-spreading under GM noise. For general case, let $\mathbf{x}_{\mathbf{i}}$ represents the ith transmitted symbol in UWA channel in presence of GM noise; the received signal will be represented as:

$$
r=x_{i}+n
$$

Wheren represents the equivalent GM noise resultant from the $\mathbf{K}$ Gaussian noise components, which has the pdf represented by (3). This received signal has a pdf of $\mathbf{f}_{\mathbf{r}}(\mathbf{n})=\mathbf{f}_{\mathbf{G M}}\left(\mathbf{n}-\mathbf{x}_{\mathbf{i}}\right)$, and thus the stochastic process of the received signal follows the same density function of the noise process with mean $\boldsymbol{\mu}_{\mathrm{rj}}=\boldsymbol{\mu}_{\mathbf{j}}+$ $\mathbf{x}_{\mathbf{i}}$, and variance $\boldsymbol{\sigma}_{\mathbf{r j}}^{2}=\boldsymbol{\sigma}_{\mathbf{j}}^{\mathbf{2}}, \forall \mathbf{j}=\mathbf{1} \ldots \mathbf{K}$ component of the mixture noise. In the BPSK case, assume that $\mathbf{x}_{\mathbf{1}}$, and $\mathbf{x}_{2}$ represent the two possible symbols have equal transmitted probability. In the non-spreading case $\mathbf{x}_{\mathbf{1}}=\sqrt{\mathbf{E}_{\mathbf{b}}}$, and $\mathbf{x}_{2}=-\sqrt{\mathbf{E}_{\mathbf{b}}}$, while after the spreading process the signal energy is attenuated by the spreading gain and $\mathbf{x}_{1}=\sqrt{\frac{\mathbf{E}_{\mathbf{b}}}{\mathbf{N}}}$, and $\mathbf{x}_{2}=-\sqrt{\frac{\mathbf{E}_{\mathbf{b}}}{\mathbf{N}}}$, where $\mathbf{E}_{\mathbf{b}}$ is the average energy per bit, and $\mathbf{N}$ is the spreading code length. The corresponding conditional pdf then can be represented in the non-spreading case as:

$$
P\left(r / x_{i}\right)=\sum_{j=1}^{K} \frac{\epsilon_{j}}{\sqrt{2 \pi} \sigma_{j}} \exp \left\{-\left(\frac{r-\left[\mu_{j}-(-1)^{i} \sqrt{E_{b}}\right.}{\sqrt{2} \sigma_{i}}\right)^{2}\right\}
$$

While in the spreading case, the conditional pdf will be represented as:

$$
P\left(r / x_{i}\right)=\sum_{j=1}^{K} \frac{\epsilon_{j}}{\sqrt{2 \pi} \sigma_{j}} \exp \left\{-\left(\frac{r-\left[\mu_{j}-(-1)^{i} \sqrt{\frac{E_{b}}{N}}\right.}{\sqrt{2} \sigma_{i}}\right)^{2}\right\}
$$

Where $\mathbf{i}=\mathbf{1}, \mathbf{2}$ determines which symbol is transmitted. Accordingly, the average error probability can be obtained, assuming equal-probable symbol transmission as:

$$
\begin{gathered}
P_{G M}^{B P S K}=\frac{1}{2} P\left(r<\frac{0}{x_{1}}\right)+\frac{1}{2} P\left(r>\frac{0}{x_{2}}\right) \\
=\sum_{j=1}^{K} \frac{\epsilon_{j}}{2 \sigma_{j} \sqrt{2 \pi}}\left\{\int_{-\infty}^{0} e^{-(r+x)^{2} / 2 \sigma_{j}^{2}} d r+\int_{0}^{\infty} e^{-(r+y)^{2} / 2 \sigma_{j}^{2}} d r\right\}
\end{gathered}
$$


Where $=-\left(\boldsymbol{\mu}_{\mathbf{j}}+\sqrt{\mathbf{E}_{\mathbf{b}}}\right), \mathbf{y}=-\left(\boldsymbol{\mu}_{\mathbf{j}}-\sqrt{\mathbf{E}_{\mathbf{b}}}\right)$ for the non-spreading case, and $\mathbf{x}=-\left(\boldsymbol{\mu}_{\mathbf{j}}+\sqrt{\frac{\mathbf{E}_{\mathbf{b}}}{\mathbf{N}}}\right), \mathbf{y}=$ $-\left(\boldsymbol{\mu}_{\mathbf{j}}-\sqrt{\frac{\mathbf{E}_{\mathbf{b}}}{\mathbf{N}}}\right)$ for the spreaded case. Assuming that $\mathbf{u}=(\mathbf{r}+\mathbf{x}) / \boldsymbol{\sigma}_{\mathbf{j}}, \mathbf{v}=(\mathbf{r}+\mathbf{y}) / \boldsymbol{\sigma}_{\mathbf{j}}$, and by making the appropriate changes of the variables, the closed form of the error probability in the non-spreading case can be obtained as:

$$
P_{G M}^{B P S K}=\sum_{j=1}^{K} \frac{\epsilon_{j}}{2}\left\{\mathcal{Q}\left(\frac{\sqrt{E_{b}}+\mu_{j}}{\sigma_{j}}\right)+\mathcal{Q}\left(\frac{\sqrt{E_{b}}-\mu_{j}}{\sigma_{j}}\right)\right\}
$$

While for the spreading case, the closed form of the error probability can be obtained as:

$$
P_{G M}^{B P S K}=\sum_{j=1}^{K} \frac{\epsilon_{j}}{2}\left\{\mathcal{Q}\left(\frac{\sqrt{\frac{E_{b}}{N}}+\mu_{j}}{\sigma_{j}}\right)+\mathcal{Q}\left(\frac{\sqrt{\frac{E_{b}}{N}}-\mu_{j}}{\sigma_{j}}\right)\right\}
$$

Where $\mathcal{Q}($.$) is the standard \mathcal{Q}$ function. It is clear that, for a single component with zero mean Gaussian process has $\boldsymbol{\epsilon}_{\mathbf{j}}=\mathbf{1}$, and $\boldsymbol{\sigma}_{\mathbf{1}}=\frac{\mathbf{N}_{\mathbf{o}}}{2}$, the relation (11) will be reduced to the ordinary expression for the probability of error of the BPSK derived in [1].

\section{III- Simulation Results}

In this section, the simulation of the UWA channel with the GM noise is presented and verified with the analytical results. The simulation is performed in the case of non-spreading case, and the spreading case with different code lengths. The spreading code considered in the simulation is a chaotic code generated from the logistic map represented by equation (12).

$$
c_{i+1}=R c_{i}\left(1-c_{i}\right), \quad c_{i} \in(0,1)
$$

Where, $\mathbf{c}_{\mathbf{i}+\mathbf{1}}$ is the new value generated from the old value $\mathbf{c}_{\mathbf{i}}, \mathbf{R}$ is the bifurcation parameter. As well known, the initial value $\mathbf{c}_{\mathbf{0}}$ affects on the rest of the generated values, which gives to the chaotic codes its main advantage of generating the huge number of the spreading sequences. The simulation is evaluated in presence of two different GM noises equivalent to two different sets of two Gaussian components; each set has the parameters shown in table II.

\begin{tabular}{|c|c|c|c|c|c|c|}
\hline NOI & \multicolumn{6}{|c|}{ GM Model Parameter } \\
\hline $\begin{array}{l}\text { DAT } \\
\text { A }\end{array}$ & $\begin{array}{l}\epsilon_{1}\left[\epsilon_{2}\right. \\
\left.=1-\epsilon_{1}\right]\end{array}$ & $\mu_{1} \mu_{2}$ & $\mu_{G M}$ & $\sigma_{1}$ & $\sigma_{2}$ & $\sigma_{G M}$ \\
\hline $\begin{array}{c}\text { SET } \\
1\end{array}$ & 0.371 & 00 & 0 & $\begin{array}{l}0.8 \\
88\end{array}$ & $\begin{array}{c}1.9 \\
3\end{array}$ & $\begin{array}{c}1.37 \\
04\end{array}$ \\
\hline $\begin{array}{c}\text { SET } \\
2\end{array}$ & 0.325 & 00 & 0 & $\begin{array}{l}0.8 \\
36\end{array}$ & $\begin{array}{c}3.1 \\
7\end{array}$ & $\begin{array}{c}1.93 \\
33\end{array}$ \\
\hline
\end{tabular}

TABLE II

PARAMETERS OF THE GAUSSIAN COMPONENTS AND THEIR EQUIVALENT GM NOISE 
Fig. 3 shows the analytical and numerical results of the BER against the SNR for the traditional BPSK without spreading in presence of traditional AWGN, and two different sets of GM noises. First of all, the figure shows that there is a great correspondence between the analytical and simulation results, which ensure that both the results are correct. The figure shows also that applying the GM noises degrades the performance by more than $2 \mathrm{~dB}$ for set 1 and $6.5 \mathrm{~dB}$ for set 2 at BER $\mathbf{1 0}^{\mathbf{- 5}}$.

In Fig. 4, the system performance is evaluated analytically and numerically in case of the spreading case, using traditional chaotic code with length 15 . The figure shows that both the analytical and numerical results are still matched in the spreading case. The results show also that, due to the spreading gain, the system performance is generally enhanced by about $12 \mathrm{~dB}$ at BER 10 ${ }^{-\mathbf{5}}$; however the GM noises still degrades the performance relative to the AWGN case.

Figs. 5 and 6 illustrate the performance when the code length is increased to be 31, and 63 respectively. The figures show that the performance improvement is increased to be about $15 \mathrm{~dB}$ and $18 \mathrm{~dB}$ at BER $\mathbf{1 0}^{-5}$ respectively. The figures show also that the simulation results verify the analytical results in the spreading case of the mentioned code lengths. It is clear also that the GM noise generally degrades the performance by about 2 to $6.5 \mathrm{~dB}$ according to the noise parameters.

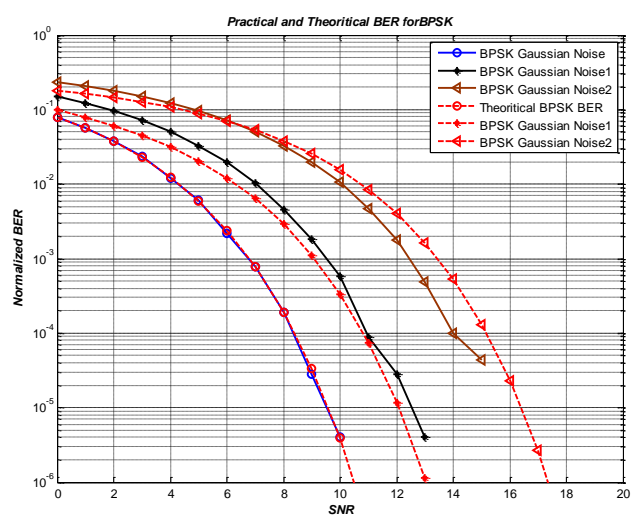

Figure 3 the analytical and numerical BER for the non-spreading case in presence of GM noise.

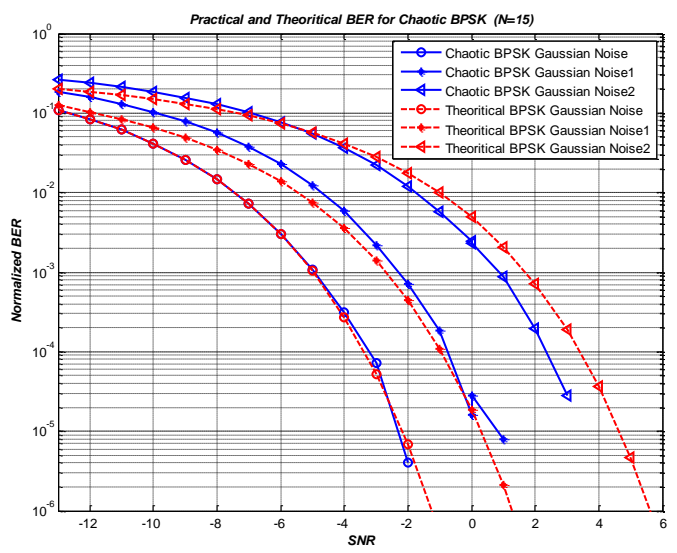

Figure 4the analytical and numerical BER for the spreading case (code length 15) in presence of GM noise

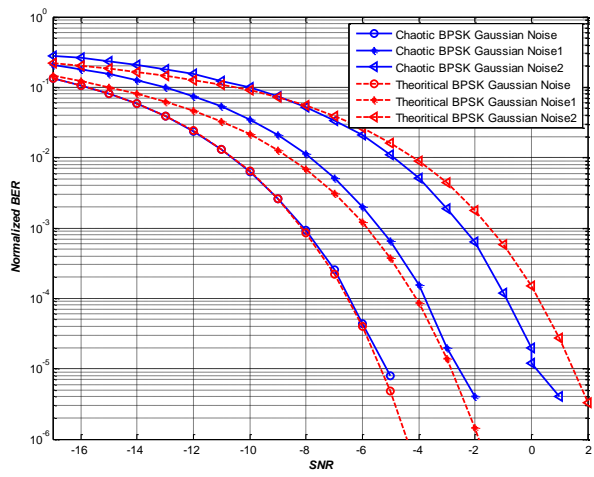

Figure 5the analytical and numerical BER for the spreading case (code length 31 ) in presence of GM noise

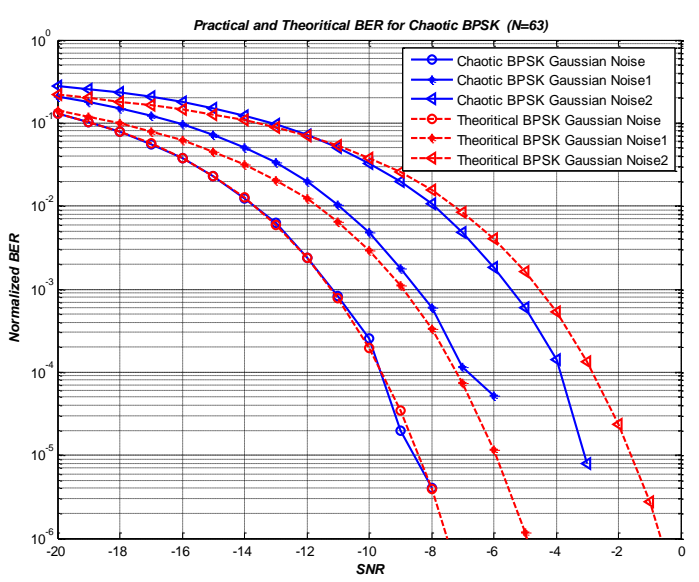

Figure 6the analytical and numerical BER for the spreading case (code length 63) in presence of GM noise 


\section{IV- Conclusion}

In this paper, an analytical analysis is presented for a CSC in presence of GM noise as a model of UWA channel. The paper begins with a brief introduction about the privileges of the chaotic codes over the PN sequences, and a brief summery about the previous work related to the GM noise. After that, the GM noise is discussed and a simple example is given. A closed form for the probability of error is derived for BPSK signal in both spreading and non-spreading cases in presence of GM noise. A simulation comparison is performed for the traditional BPSK in the spreading and non-spreading cases in presences of AWGN and GM noises to verify the analytical results. The applied spreading code is the traditional chaotic code, with different code lengths. Two different scenarios are proposed to simulate the GM noise as an equivalent to two different components of Gaussian noises. The results show that the GM noise generally degrades the performance of the BPSK. The results show also that the analytical and the numerical results are completely matched. According to the results, applying the CSC improves significantly the system performance in presence of the GM noise according to the code length. The logistic and tent maps have nearly close initial values in presence of the AWGN channel, whereas in fading channels the initials have different values. The results show also that most of the enhancement operations have positive effect on the statistical properties when the initial values are optimized. The performance evaluation show that the traditional and ZM logistic codes has better performance compared with the Gold code in presence of FSF channel. In Relight fading channel the most of the logistic codes outperformed the performance of the Gold code. For the tent map, the ZM process significantly improves the performance of the ZM, and SBZM codes over the Gold code.

\section{References}

[1] Henrieta P, "The type of chaotic sequences for signal transmission", SCYR - 10th Scientific Conference of Young Researchers - FEI TU of Košice 2010.

[2] R. Vali, S. Berber, and S. K. Nguang, "Accurate derivation of chaos-based acquisition performance in a fading channel”, IEEE Trans. Wireless Commun., vol. 11, no. 2, pp. 722-731, Feb 2012.

[3] G. Kaddoum, D. Roviras, P. Charge, D. Fournier-Prunaret, "Performance of multi-user chaos-based DS-CDMA System over multipath channel” IEEE Trans, 2009.

[4] Proakis, J. G., Digital Communications, 4thed, McGraw-Hill, NY, 2000.

[5] LANGARD, LISE, et al. "Sound production in pre-spawning herring, cod and haddock in a naturally enclosed ecosystem." Bioacoustics 17.1- 3 (2008): 38-40.

[6] Dahl, Peter H., et al. "Underwater ambient noise." Acoustics Today 3.1 (2007): 23-33.

[7] Hildebrand, John A. "Anthropogenic and natural sources of ambient noise in the ocean." Marine Ecology Progress Series 395.5 (2009).

[8] W. M. Carey, Ocean Ambient Noise, Springer, 2011.

[9] Banerjee, Sharbari. Agrawal, Monika; "Underwater Acoustic Noise with Generalized Gaussian Statistics: Effects on Error Performance," OCEANS, 2013 - Bergen , 10-13 June 2013

[10]Middleton, D., "Non-Gaussian noise models in signal processing for telecommunications: new methods and results for class A and class B noise models," Information Theory, IEEE Transactions on, vol.45, no.4, pp.1129-1149, May 1999.

[11]Nadarajah, Saralees. "A generalized normal distribution." Journal of Applied Statistics, vol. 32, no. 7 (2005): 685-694.

[12]C. L. Nikias and M. Shao, Signal processing with alpha-stable distributions and applications, New York, NY, USA, 1995. 
[13]Swami, A., "Non-Gaussian mixture models for detection and estimation in heavy-tailed noise," Acoustics, Speech, and Signal Processing, 2000. ICASSP '00. Proceedings. 2000 IEEE International Conference on , vol.6, no., pp.3802,3805 vol.6, 2000.

[14]Stein, D.W.J.;, "Detection of random signals in Gaussian mixture noise," Information Theory, IEEE Transactions on , vol.41, no.6, pp.1788-1801, Nov 1995.

[15]Douglas A. Reynolds, "Gaussian Mixture Models," Encyclopedia of Biometrics, pp. 659-663, 2009.

[16]Senol, Habib, et al. "Channel estimation in underwater cooperative OFDM system with amplify-andforward relaying." Global Communications Conference (GLOBECOM), 2012 IEEE. IEEE, 2012.

[17]Madadi, Z.; Anand, G.V.; Premkumar, A.B., "3-D source localization in shallow ocean with nonGaussian noise using a linear array of acoustic vector sensors," Information Science, Signal Processing and their Applications (ISSPA), 2012 11th International Conference on , vol., no., pp.1353,1358, 2-5 July 2012

[18]Bo Zhao; Blanke, M.; Skjetne, R., "Particle filter ROV navigation using hydroacoustic position and speed log measurements," American Control Conference (ACC), 2012, vol., no., pp.6209,6215, 27-29 June 2012 